\title{
DOS MIRIDOS NUEVOS DE LA PROVINCIA DE ALICANTE \\ (Insecta, Heteroptera)
}

\author{
Por J. RIBES
}

SUMARIO

Página

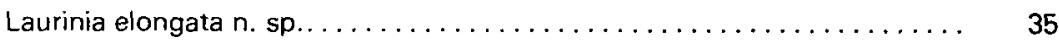

Compsidolon (Apsinthophylus) Sauledai n. sp................ 42

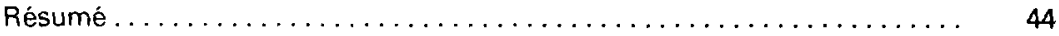

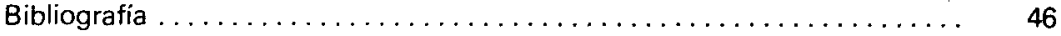



Macho: tamaño mediano. Muy alargado: 4,3 veces más largo que la anchura del pronoto (Fig. 1). Coloración típica del género; brillante.

Cabeza corta, rugosa, de color ferrugíneo oscuro, 2,43 veces más ancha que larga. Ojos redondeados, rebasando por detrás el borde posterior de la cabeza, en forma de listón. Relación sinlipsis/ojo $=1,4$. Antenas recubiertas de pelos cortos y delgados, blancos. Primer artejo de las mismas cilíndrico, estrechado por el centro, con la base ferrugínea y el resto de un amarillo sucio. Los otros tres, algo curvados hacia afuera, son pardo oscuros. El segundo y el tercero aparecen más delgados en la base que en el ápice. El cuarto es fusiforme. Tercero y cuarto artejos 1,27 tan largos como el segundo. Proporción de los cuatro artejos $=9: 42,5: 28: 26$ (Fig. 2). Pico negruzco, sin sobrepasar las coxas intermedias; su primer artejo, muy grueso, rebasa apenas el borde posterior de la cabeza.

Pronoto claramente dividido en una parte anterior, en forma de rodete rugoso, débilmente bilobulado, ferrugineo y otra posterior, globosa, negra, recubierta de pelos blancos, cortos, poco densos. Es 1,41 más ancho que la diátone (=máxima anchura de la cabeza) y a su vez el segundo artejo de las antenas es 0,88 tan largo como la anchura mayor del pronoto.

Escudete negro, muy convexo, redondeado por detrás y con un surco mediano en su tercio anterior. Aparece recubierto por el mismo tipo de pilosidad que el pronoto.

Hemélitros muy largos, sobrepasando ampliamente el extremo del abdomen, con las seis manchas laterales y las dos del clavus, todas marfileñas, dispuestas parecidamente a las de Laurinia fugax Rt. (Figura 4). Las dos manchas intermedias, sin embargo, ocupan sólo la exocoria, no adentrándose en la mesocoria, como ocurre en la especie tipo del género. Dicha mesocoria presenta, además, una coloración rosada granulosa con fondo amarillo, mientras la exocoria, clavus y cúneo tienen un color ferrugíneo oscuro. La membrana es uniformemente negruzca, apenas brillante; en cambio el área de las nerviaciones de la misma es muy brillante, al igual que el cúneo. La pilosidad de los hemélitros comprende elementos enderezados, largos, oscuros, diseminados y otros 
algo más cortos y blanquecinos; los primeros distribuidos sobre todo por el centro, los segundos por los lados. Hay además pelos escamiformes plateados entremezclados con los anteriores.

Patas de un ferrugíneo oscuro. Fémures con el ápice amarillento, provistos de pelos blanquecinos, cortos, diseminados; los fémures posteriores están algo engrosados en su parte anteapical. Tibias con un anillo preapical amarillento, recubiertas de pilosidad blanca más densa y larga en sus tercios distales; la parte interna de éstos está recorrida asimismo por espinas claras en la base y ferrugíneas en su mitad libre. Apice de las tibias posteriores provisto de una corona de espinas robustas, ferrugíneas, envolviendo la base del primer artejo del tarso, que es de la misma longitud que el tercero. El segundo artejo es de 0,76 tan largo como cualquiera de los otros dos. Las uñas son menos curvadas que en L. fugax Rt., con los pseudoarolios poco visibles y los arolios gruesos, convergentes como en ésta (Fig. 3 ).

La parte ventral es negruzca y los segmentos abdominales aparecen recubiertos de pelos blanquecinos, poco densos.

Pigóforo triangular, con la escotadura dorsal muy abierta y la ventral en forma de $J$ invertida; su borde izquierdo es casi recto (Figs. 5 y 6). Parámero izquierdo muy largo, arqueado, provisto de una hipófisis aguzada por la parte distal y con otra espina proximal muy robusta y puntiaguda; el tubérculo sensorial es triangular y los largos pelos sensitivos, en número de cuatro, emergen de su borde posterior, lejos de la punta (Fig. 7). Pene alargado, dispuesto como muestra la fig. 9, con la vesica larga y estrecha $y$ las dos estructuras quitinizadas de la misma soldadas en forma de colmillo (Fig. 8).

Long.: macho $=5,7 \mathrm{~mm}$; hembra $=$ desconocida.

Discusión: El género Laurinia Rt. hasta ahora era monotípico. Su especie L. fugax fue descrita por REUTER de los alrededores de Túnez (1884), aunque posteriormente se encontró en Marruecos (1940), Argelia (1943) y en Aranjuez (Madrid) (1957). Debo añadir otra cita mía - una hembra - no publicada de la Serra d'Ancosa, cerca de Pontons (Barcelona) (30-VI-1973). L. elongata n. sp. (macho) es mucho más larga que L. fugax Rt. (macho); ésta es 3,3-3,4 mayor que la anchura del pronoto. La relación sinlipsis/ojo en L. fugax Rt. es 1,8 (2,0 según WAGNER), mientras que en la nueva especie es 1.4. El tercero y cuarto artejo de las antenas son, en L. fugax Rt. sólo 1,15 tan largos como el segundo, al tiempo que en $L$. elongata $n$. sp., la relación es de 1,27. La pilosidad del pronoto de L. fugax Rt. es muy densa y el escudete carece de surco 
transverso. La mesocoria rosada granulosa con fondo amarillo y la mancha intermedia reducida a la exocoria caracterizan a L. elongata $n$. sp. El borde izquierdo del pigóforo convexo y la abertura dorsal cerrada (Figuras 10 y 11), el parámetro izquierdo más corto con la hipófisis sólo con una pequeña espina proximal y el tubérculo sensorial corto, robusto, provisto de cinco pelos sensitivos emergiendo de su ápice (Fig. 12), así como las estructuras quintinizadas de la vesica del pene (Fig. 13) son caracteres propios de la especie de REUTER que permiten una fácil separación de L. elongata $\mathrm{n}$. $\mathrm{sp}$.

Material: Un macho, de Alicante, Arenals del Sol, 27-IV-75; en la playa. N. Sauleda leg. Holotipo en mi colección. 


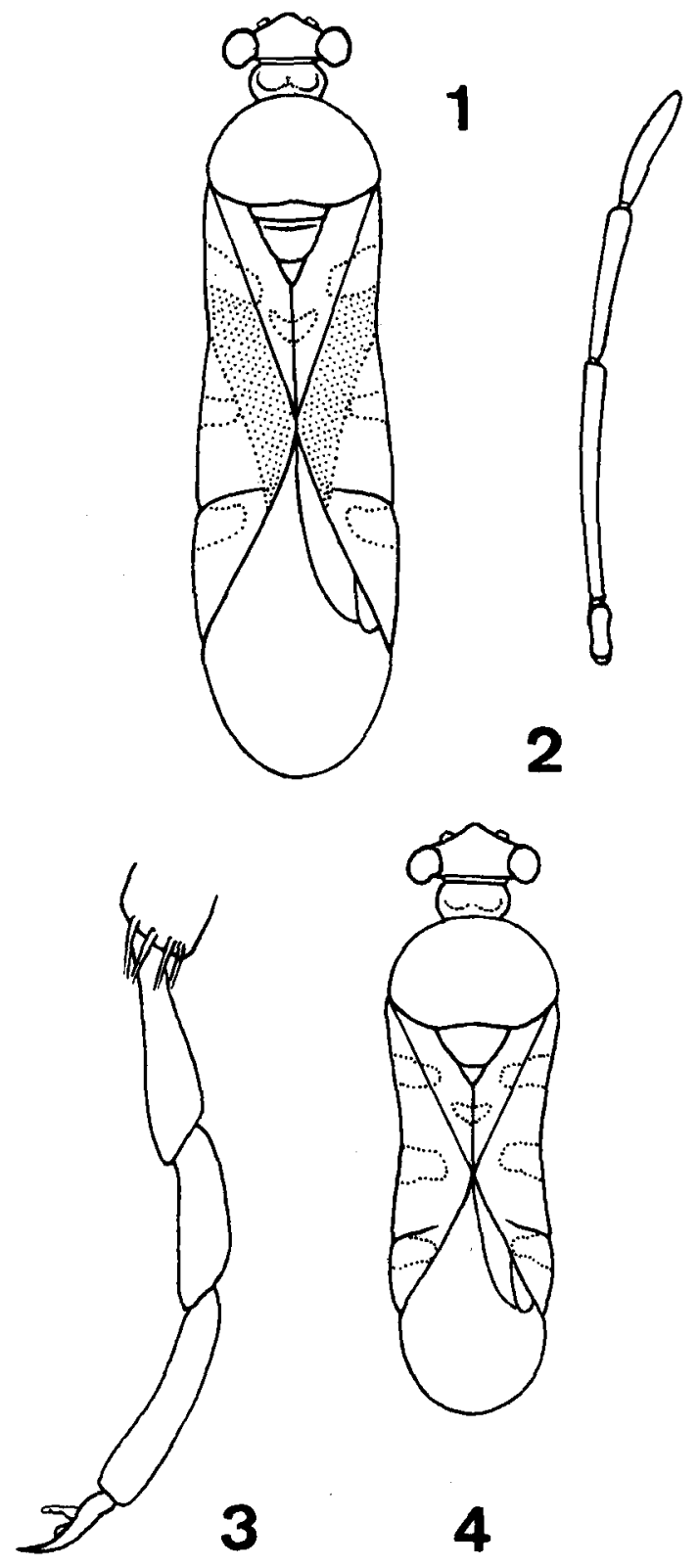

FIGS. 1 a 4. Fig. 1: Laurinia elongata n. sp. macho. Fig. 2: Su antena. Fig. 3: Su tarso posterior. Fig. 4: Laurinia fugax Rt. macho. 


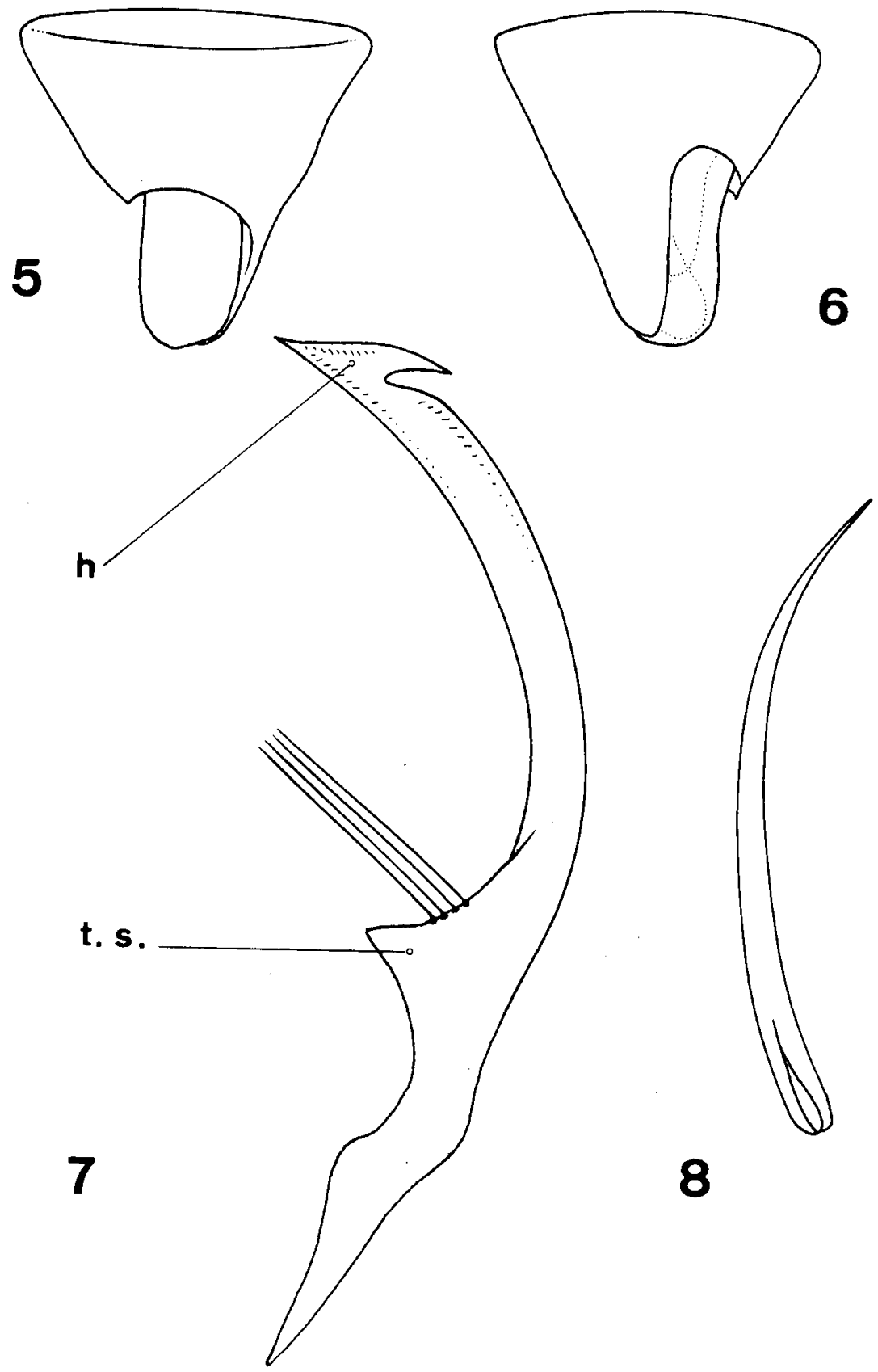

FIGS. 5 a 8. Laurinia elongata n. sp. Fig. 5: Pigóforo, por dorsal. Fig. 6: Pigóforo, por ventral. Fig. 7: Parámero izquierdo; h: hipófisis; t.s.: tubérculo sensorial. Fig. 8: Estructuras quitinizadas de la vesica del pene. 


$$
\text { . }
$$




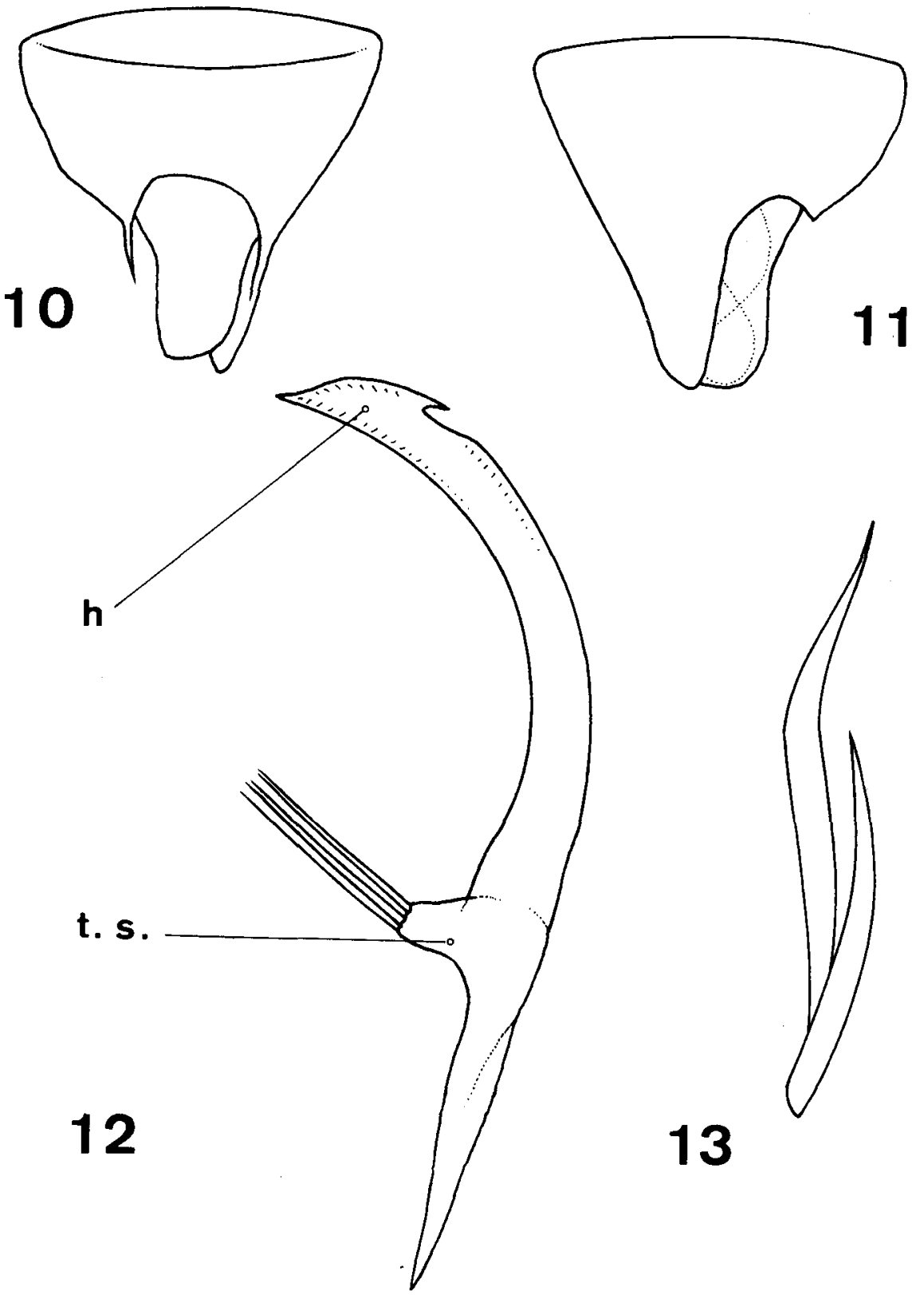

FIGS. 10 a 13. Laurinia fugax Rt. Fig. 10. Pigóforo, por dorsal. Fig. 11: Pigóforo, por ventral. Fig. 12: Parámero izquierdo; h: hipófisis; t.s.: tubérculo sensorial. Fig. 13: Estructuras quitinizadas de la vesica del pene. 
Tamaño pequeño. Coloración general amarilla verdosa. Brillante. Dorso recubierto de pelos semiinclinados e inclinados, dorados, brillantes, muy abundantes; en la parte anterior, especialmente, algo oscurecidos. Puntuación dorsal ferrugínea, con elementos finos $y^{\prime}$ regulares. Dimorfo. Macho: Contorno más de dos veces más largo que ancho; bordes elitrales paralelos. Hembra: Contorno menos de dos veces más largo que ancho; ovoidal, bordes elitrales convexos.

Cabeza corta, 1,25 (macho) ó 1,30 (hembra) más ancha que alta (Figs. 14 y 15). Tilus grueso, con los bordes algo cóncavos, más ancho en su tercio apical y con la punta redondeada. Genas más o menos enrojecidas. Frente ancha con 6-8 arcos ferrugíneos por lado, poco marcados y puntuación ferrugínea marginal y superior. Ojos medianos de color rosado o rojizo, globosos; relación sinlipsis/ojo $=1,8-1,9$ (macho) y $2,3-2,4$ (hembra). Antenas amarillentas, excepto el primer artejo, oscurecido por lo menos en sus dos tercios basales; recubiertas de pelos densos, cortos, oscurecidos; no presentan apenas dimorfismo, siendo la proporción de los cuatro artejos $=5: 20: 15,5: 7,5$ (macho) y $5: 17,5$ : $14: 7$ (hembra) (Figs. 16 y 17). El segundo artejo es 1,34 (macho) ó 1,10 (hembra) tan largo como la diátone y 0,93 (macho) ó 0,82 (hembra) tanto como el borde posterior del pronoto. Pico muy largo, amarillento, con los dos tercios basales del prinner artejo, zonas articulares y mitad distal del último artejo más o menos oscurecidos; alcanza el cuarto segmento abdominal.

Pronoto trapezoidal, 2,6-2,7 más largo que ancho; su lóbulo anterior más o menos oscurecido o enrojєcido en el macho; el borde posterior es 1,42 (macho) ó 1,34 (hembra) tan largo como la diátone.

Escudete triangular, oscurecido en el macho, con las callosidades de los ángulos anteriores amarillas; 1,5 (Macho) ó 1,6 (hembra) más ancho que largo.

Hemélitros sobrepasando ampliamente el extremo del abdomen (macho) o sin rebasarlo (hembra). Membranas blancas, salpicadas abundantemente de gris oscuro en toda su superficie, lo que les da un aspecto finamente moteado. Nerviaciones amarillentas. 
Patas recubiertas de una pilosidad algo oscurecida. Fémures ferrugíneos con irroración rojiza, los posteriores muy ensanchados (macho y hembra) con su ápice provisto de dos callosidades - dorsal y ventralredondeadas, amarillentas, brillantes. Tibias amarillentas, las posteriores con el borde interno enrojecido, provistas, además de la pilosidad general, de sedas largas, algo oscurecidas, inclinadas unos cuarenta y cinco grados, emergiendo de puntos ferrugíneos muy gruesos. Tarsos amarillentos con el ápice del tercer artejo y uñas negruzcos. Segundo artejo algo mayor que el tercero. Proporción de los tres artejos $=8: 16: 15$. Uñas alargadas sólo curvadas ligeramente en el ápice; pseudoarolios muy pequeños y cortos, siendo ampliamente sobrepasados por las sedas aroliares que alcanzan casi la mitad de la uña (Fig. 18).

Parte inferior del tórax ferrugínea. Segmentos abdominales amarillos teñidos más o menos de verde, recubiertos de una pilosidad dorada, brillante.

Pigóforo cónico, 1,5 más largo que ancho; su borde derecho es cóncavo y la escotadura genital en semióvalo alargado (Fig. 19). Parámero derecho simple, en forma de hoja elíptica terminada en una hipófisis muy corta y algo inclinada (Fig. 20). Parámero izquierdo en tenaza, con la hipófisis hacia arriba, aguzada y el tubérculo sensorial ancho, laminar, terminado en punta hacia abajo (Figs. 21 y 22). Vesica del pene simple, en forma de $\mathrm{J}$, delgada, con el ápice no bifurcado, corto, algo inclinado; el gonóporo secundario es alargado y subapical (Fig. 24). Teca robusta, puntiaguda, con la escotadura que muestra la fig. 23.

$$
\text { Long.: macho }=3,0-3,3 \mathrm{~mm} \text {, hembra }=2,8-3,0 \mathrm{~mm} \text {. }
$$

Discusión: Por la puntuación dorsal regular y marcada, el segundo artejo de las antenas más corto que el pronoto, la proporción de los artejos segundo y tercero de los tarsos posteriores y sobre todo por la estructura, delgada y sin bifurcación apical, de la vesica del pene Compsidolon sauledai $\mathrm{n}$. sp. debe integrarse en el subgénero Apsinthophy/us Wgn. La coloración dorsal amarilla verdosa, el pigóforo alargado y la forma en $J$ de la vesica del pene lo separan de las demás especies del subgénero. El primer artejo de las antenas oscurecido lo aleja de $C$. absinthii (Sc.) (holomediterráneo), C. saxosus Ptsh. (Kirguizistán), C. eckerleini Wgn. (Tunicia), C. nanno Lv. (Lérida, comarca del Segrià) y C. saundersi (Rt.) (Argelia, Libia). C. beduinum Lv (Egipto) y C. minutum Wgn. (Argelia) miden menos de $2,6 \mathrm{~mm}$; por cierto esta última especie descrita y citada sólo de Tassili n'Ajjer la he capturado en EI Pinetell (Tarragona), 21-VII-1973 (WAGNER det!). El macho de C. alc- 
mene Iv. (Irán) rebasa los 4,2 mm., la relación sinlipsis/ojo es 1,4 y tiene el cúneo algo enrojecido. La ćabeza y parte anterior del pronoto de C. scutellare (Rt.) (Marruecos, Argelia) son negruzcos. La coloración de la membrana y los artejos tarsales segundo y tercero iguales de $C$. pumilum (Jak.) (holomediterráneo) lo separan de C. sauledai n. sp. Las nerviaciones de la membrana oscuras caracterizan a $C$. atomosum (Rt.) (pontomediterráneo). C. balachowsky (Wgn.) (España, N. de Africa) y C. ribesi Lv. (Murcia, Totana) tienen las superficies de las membranas de un gris oscuro con zonas más claras, pero nunca finamente moteadas; a esta última especie hay que añadir otra cita de Novelda (Alicante), 18-I-1975 (Sauleda leg., Ribes det.).

Material. Cuatro machos y seis hembras de la Laguna de Salinas (Alicante); 2-VI-73, 4-VII-73 y 1-VIII-73. Sobre Suaeda splendens Gr. \& G., N. Sauleda leg. Holotipo (un macho) en mi colección; paratipos también en mi colección y en la de N. Sauleda.

Con gran placer dedico esta interesante especie a mi amigo N. Sauleda, cuya metódica prospección de la zona alicantina ha logrado hallazgos sorprendentes que serán objeto de un posterior trabajo.

$R \dot{E} S \cup M \dot{E}$

On décrit ici deux espèces de Miridae de la province d'Alicante. Laurinia elongata n. sp., mâle, s'écarte de L. fugax Rt., jusqu'alors une espèce monotypique, par son corps plus allongé, le rapport synlipsis/oeil inférieur $(1,4)$, le pronotum à pilosité faible et éparse, la mésocorie granuleuse rosée à fond jaune, le bord gauche du pygophore presque droit et, enfin, le paramère gauche très long pourvu d'une hypophyse avec deux épines bien developpées ainsi que d'un tubercule sensoriel triangulaire à quatre poils sensitifs sortant loin de son extremité. Compsidolon (Apsinthophy/us) sauledai n. sp. est la seule espéce du sous-genre, parmi la douzaine qu'il renfermait, à coloration dorsale jaune verdâtre, le pygophore extrêmement allongé aussi bien qu'avec la forme simplifiée, en $\mathrm{J}$, de l'ampoule du pénis. 


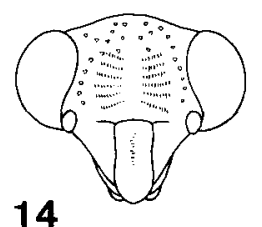

14

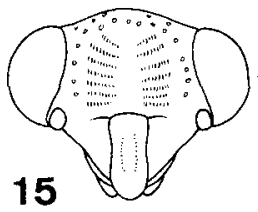

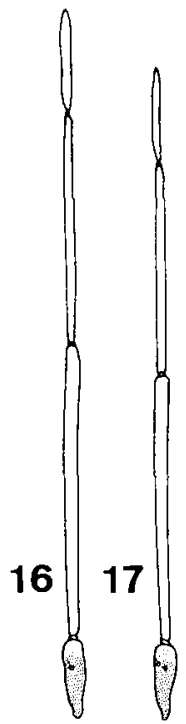
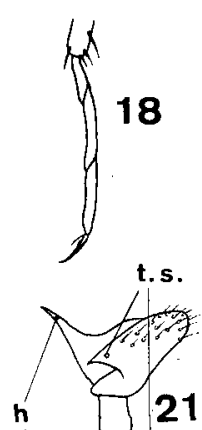

21

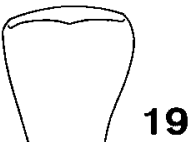

$19 \quad 23$
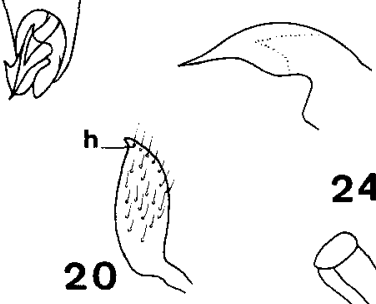

24
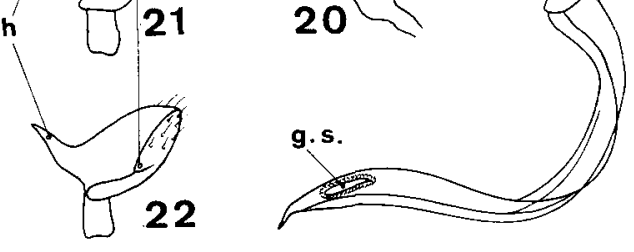

FIGS. 14 a 24. Compsidolon (Apsinthophylus) sauledai n, sp. Fig. 14: Cabeza del macho, de frente. Fig. 15: Cabeza de la hembra, de frente. Fig. 16: Antena del macho. Fig. 17: Antena de la hembra. Figura 18: Tarso posterior del macho. Fig. 19: Pigóforo, por dorsal. Fig. 20: Parámero derecho; h: Hipófisis. Figs. 21 y 22: Parámero izquierdo en dos posiciones; h: hipófisis; t.s.: tubérculo sensorial. Fig. 23: Teca. Fig. 24: Vesica del pene; g.s.: gonóporo secundario. 
LINDBERG, H., 1940. - Inventa entomológica itineris Hispanici et Maroccani. Die Capsiden-fauna von Marokko, Soc. Sc. Fenn., Comm. Biol. VII (14), ps. 1-55.

LINDBERG, H., 1956. - Uber einige Miriden in E. de Bergevins Sammlug, Not. Ent. $X X X V I$, ps. 53-64.

LINNAVUORI, R., 1971. - On some new or little known Miridae species, Misc. Zool. V. III. fasc. 1, ps. 27-33.

LINNAVUORI, R., 1971. - A new species of the genus Compsidolon Rt. from Iran (Het Miridae), Stutt. Beitr. z. Naturk, N. ${ }^{\circ} 227$, ps. 1-4.

LINNAVUORI, R., 1972.-A new species of the genus Compsidolon Rt. (Het Miridae) from Spain, P. Inst. Biol. Apl. V, 52, ps. 125-127

PUTSHKOV, V. G., 1975. - Nuevas especies de míridos de la fauna del sur del Kirguizistán (Het.) (en ruso, resumen en inglés), Dokl. Akad. Nauk. Ukr. SSR, Kiev, Ser. B, núm. 4 , ps. 377-380.

REUTER, O. M., 1884. - In FERRARI, P. M.: Materiali per lo studio della fauna tunisina racconti da G. e L. Doria, V. Rincoti, Ann. Mus. Civ. St. Nat. Genova, XXI, Ser. 2. ${ }^{a}, \mathrm{VI}$, ps. $481-482$.

WAGNER, E., 1957. - Laurinia Reuter, 1884, und Formicocoris Lindberg, 1940 (Hem. Het. Miridae). Deut. Ent. Zeit. Neue Folge Band 4 (V), ps. 263-267.

WAGNER, E., 1965. - Die Gattung Compsidolon Reuter, 1889 (Heteroptera Miridae). Not. Ent. XLV, ps. 113-137.

WAGNER, E., 1970. - Einige neue Miriden aus Algerien und Tunesien (Hem. Het.). Reichenbachia, V, 12 , núm. 29 , ps. $287-293$.

WAGNER, E., 1973. - Die Miridae Hahn, 1831, des Mittelmeerraumes und der Makarroneschen Inselm (Hemiptera, Heteroptera). Ent. Abhand, V, 39, Supl. Parte 2. ${ }^{a}$, ps. 113 y $289-290$. 